\title{
La carte, le géographe et le cartographe
}

\section{Georges Roques}

\section{(2) OpenEdition}

\section{Journals}

Édition électronique

URL : http://journals.openedition.org/trema/2429

DOI : $10.4000 /$ trema.2429

ISSN : 2107-0997

\section{Éditeur}

Faculté d'Éducation de l'université de Montpellier

\section{Édition imprimée}

Date de publication : 1 mai 1992

Pagination : 41-46

ISSN : 1167-315X

\section{Référence électronique}

Georges Roques, «La carte, le géographe et le cartographe », Tréma [En ligne], 1 | 1992, mis en ligne le 02 décembre 2013, consulté le 30 avril 2019. URL : http://journals.openedition.org/trema/2429 ; DOI : 10.4000/trema.2429

Ce document a été généré automatiquement le 30 avril 2019

Trema 


\section{La carte, le géographe et le cartographe}

\section{Georges Roques}

1 La carte est un outil, outillage mental, construction de l'esprit. Elle peut devenir artefact. Elle n'est ni plus ni moins neutre que d'autres supports. Elle est un des éléments des représentations multiples en géographie, rien que cela, mais tout cela.

2 Artefact : phénomène d'origine humaine qui ne doit pas être artificiel à l'excès.

3 Elle est partie intégrante du raisonnement géographique qui ne peut exister valablement sans référence à la surface du globe. C'est une forme graphique qui exprime par un dessin, traits et plages, des relations. La carte est une des représentations possibles, majeure mais non exclusive.

4 Carte : représentation graphique plane de phénomènes localisables dans l'espace.

5 L'idée principale est double :

- si la carte est nécessaire, et elle l'est, elle n'est pas suffisante ;

- les représentations spatiales sont au cœur de la démarche géographique

6 Démarche : "L'examen attentif d'une configuration spatiale (forme) met sur la piste d'hypothèses (induction). L'information générale dont on dispose sur la position géographique et sur la valeur de l'espace considéré met aussi sur la piste d'hypothèses (déduction). La recherche expérimentale consiste en un certain nombre d'allers et retours et d'ajustements successifs dans les deux sens » Roger Brunet

7 Il faut redonner du sens à l'image spatiale, la faire, la défaire, la refaire, en faire d'autres sur le même espace et à d'autres échelles, la comprendre, l'animer et la faire vivre, pour soi et les autres.

8 Partant de là, on dessinera à gros traits les compétences nécessaires à acquérir, non pas pour faire des cartes, c'est l'affaire des cartographes ou des établissements spécialisés comme l'IGN, mais pour enseigner la géographie aux élèves. 


\section{Alors pourquoi les cartes?}

9 Il faut dire ce qu'elles sont, se souvenir de ce qu'elles ont été, imaginer ce qu'elles pourraient être, réfléchir à ce qu'elles montrent, découvrir ce qu'elles cachent, rêver aux espaces et à ceux qui nous les montrent.

10 Il est bon d'abord de préciser et d'élargir ce que l'on entend par cette déclaration d'intention. Faire soigneusement la différence entre croquis, trop souvent de synthèse (avec le croquis régional qui en fut la quintessence), schéma, simplification de la réalité, carte thématique, distribution d'un phénomène sur l'espace et modèles et chorèmes, qui ont leur propre logique de construction systématique et spatiale. Tous ont un langage. De la rigueur et de la pertinence du choix dépendent la qualité et la transparence du message transmis (et reçu?).

11 Modèles : représentation épurée de la réalité par une forme spatiale. Permet ainsi d'en saisir les structures et d'en montrer le fonctionnement.

Chorèmes : structures élémentaires de l'espace pouvant être représentées par un modèle graphique. Leur combinaison fait modèle.

13 Il faut ensuite dire à quoi elles servent. La carte n'est pas seulement un élément de repérage spatial, elle est un moyen de compréhension des répartitions des activités des sociétés à la surface de la terre. En ce sens, elle est partie intégrante d'une heuristique. Elle ne peut être juxtaposée, collée à la démonstration. Elle n'est pas obligatoirement une fin en soi. Elle est réflexion et démonstration avant d'être communication. L'oublier est se mettre en porte à faux de la démarche géographique, et prendre pour une fin ce qui n'est qu'un moyen.

14 Dans le meilleur des cas, les cartes servent à chercher, à démontrer et à communiquer. $\mathrm{A}$ chaque stade son style de cartographie, de charte graphique, sans que l'un soit systématiquement meilleur que les autres.

15 Heuristique : art de trouver, s'appuyant sur l'herméneutique; art d'interpréter, sans devenir hermétique. 


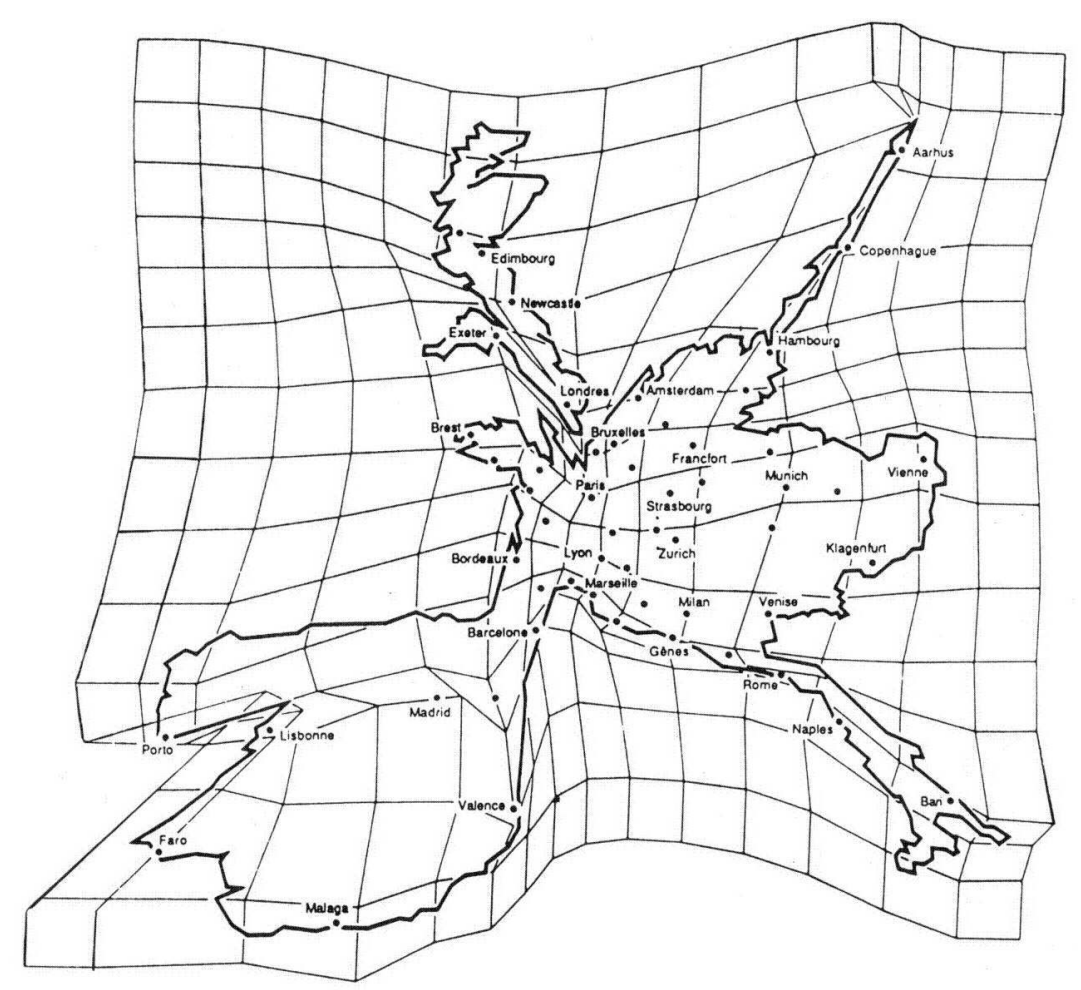

\section{Le géographe dispose d'outils divers et complémentaires}

Si la géographie s'appuie sur l'analyse de système, elle se doit d'en pratiquer les outils et les méthodes, en adaptant à son objet un paradigme venu d'autres sciences. Un des fondements de ces approches, c'est le fait que pour perdurer, et être observables, les systèmes doivent toujours se rééquilibrer par des boucles de rétroactions. C'est leur finalité essentielle. Ces boucles ont des processus simultanés, et non successifs. L'utilisation du plan permet de respecter cette simultanéité, ce que n'autorisent ni le texte écrit ni le discours.

Système : Ensemble d'éléments en interrelation dynamique. (Bertalanffy).

Les graphes sagittaux, les arbres logiques, les histogrammes, diagrammes et cartogrammes, et idéalement les systèmes d'information géographiques (S.I.G.) s'accomodent fort bien des surfaces, tout aussi bien que le récit qui sied à l'historien pour privilégier le temps, les successions, les irréversibilités.

Histogramme : répartition des valeurs d'une variable.

SIG : Système automatisé d'enregistrement et d'analyse des données permettant de traiter des données géographiques référencées et les attributs qui s'y rapportent. 


\section{Leur usage oblige à quelque rigueur}

21 Ces représentations visent non seulement à décrire, mais surtout à expliquer, à décoder le réel, chacun ayant le sien, le concret, qui n'en est que la partie émergente privilégiée par la perception de chacun. Il n'est jamais donné en lui même. Il n'est saisi qu'au travers de l'idéal et du schématique. et sera donc à facettes. Dans sa vie professionnelle, il a plusieurs avatars : il fait cours en classe, il prépare ses leçons, il peut être actif dans des clubs vidéos ou informatique... Il y a cependant une base commune, ce que chacun doit a minima maitriser, et une base optimiste que certains devraient viser, en complémentarité des compétences des collègues proches. décomposition, de reconstruction, de décodage et de décryptage des représentations graphiques, pour pouvoir utiliser à bon escient la multitude des cartes produites. Il leur faut aussi une bonne culture spatiale pour avoir une attitude critique vis à vis du corpus documentaire mis à leur disposition, qui peut plus cacher, déformer que montrer.

Ils doivent aussi faire des cartes, ce qui ne signifie pas faire de la copie ou du dessin, avec une contrainte forte qui est celle de la faiblesse des équipements, en particulier informatiques des établissements. Un langage graphique adapté à des contingences fortes, mais scientifiquement établi permet de pallier à cette lacune. 

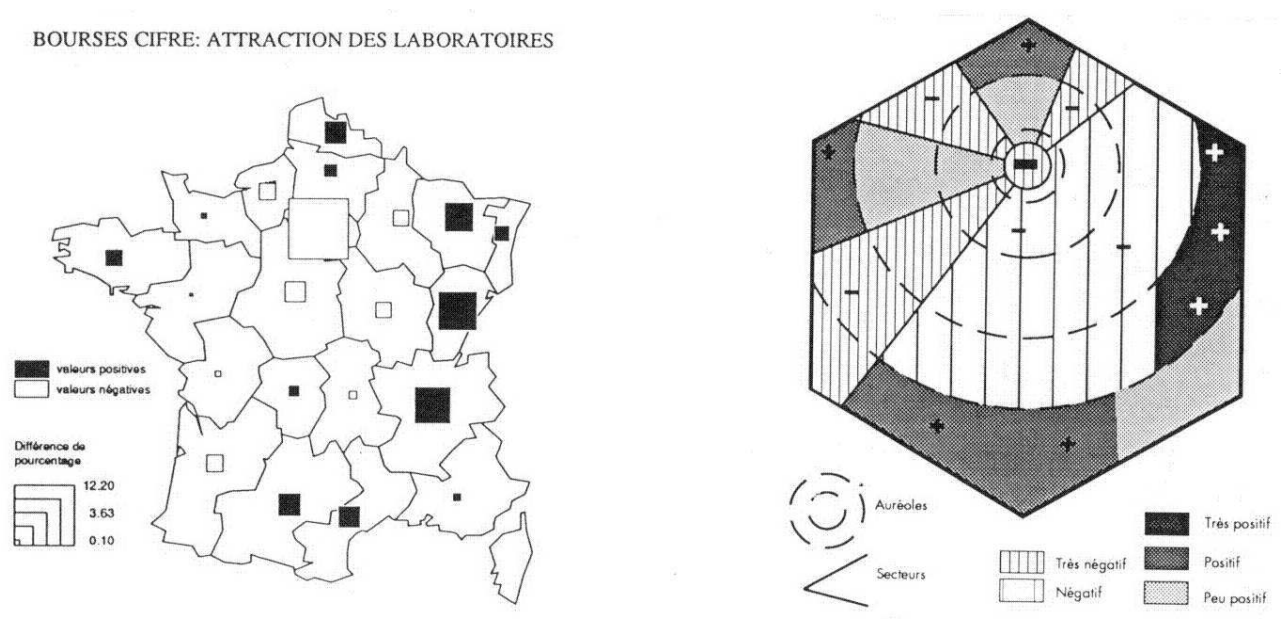

Les enseignants géographes devraient connaître les lois d'organisation de l'espace, les règles de la sémiologie graphique, devraient savoir traiter quelques données (ordinales, qualitatives, en valeur absolue, relatives). On pourrait attendre qu'ils maîtrisent les traitements statistiques simples, certaines analyses multivariées, les types de discrétisation (classes définies, d'égale importance, à effectifs égaux, écart-type...).

Discrétisation : règles permettant la répartition en classes d'une variable dans une série statistique. Séparer, classer en fonction de ce que l'on cherche ou souhaite montrer.

Certains d'entre eux, selon leurs intérêts propres, peuvent fabriquer eux-mêmes des documents, cartes, modèles, diagrammes. On peut aller jusqu'à une notion d'équipe, idéalement d'établissement, où un enseignant aurait particulièrement la charge... et les moyens de cette dimension spatiale.

Pour quelques-uns, la maîtrise de plusieurs logiciels, de maniement relativement facile, permet la réalisation de cartes. L'animation, la dynamique des cartes par l'image vidéo, les images fictives, sont des pistes intéressantes qu'il faudra explorer mieux, et plus intensément. Les supports optiques, CD ROM, CDI, vidéodisques ouvrent de fortes perspectives à des parcours différenciés et individualisés, riches en complémentarités multiples et en ouverture sur la vie.

\section{BIBLIOGRAPHIE}

AURIAC F., (1983), Système économique et espace. Paris, Economica, 216 p.

BAREL Y., L'idée de système dans les sciences sociales, Esprit, $\mathrm{n}^{\circ} 1$.

BERTIN J., (1967), Sémiologie graphique, Paris, Mouton et Gauthier-Villars

BRUNET R., (1987), La Carte, mode d'emploi, Paris, Fayard / RECLUS, 269 p.

ClARY M., DUfAU G., DURAND, FERRAS R., (1987), Cartes et modèles à l'école, Montpellier, 112 p. 
CHARRE J., MIELLET Ph., WANIEZ Ph., (1991), Pratique des systèmes d'information géographique raster. Collection RECLUS mode d'emploi, Montpellier. $50 \mathrm{p}$.

DOLLFUS O., BRUNET R., (1990), Mondes nouveaux, t. 1 de la Géographie Universelle, Paris / Montpellier, Hachette/RECLUS, 554 P.

JOLY F., (1985), La cartographie, PUF, (collection Q.S.J.)

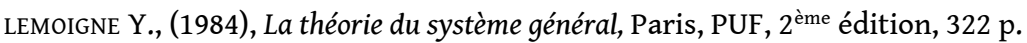

LEMOIGNE Y., (1984), la modélisation des systèmes complexes, Paris, Dunod, 180 p.

von BERTALANFFy L., (1973), Théorie générale des systèmes, Paris, Dunod, 296 p.

SANDERS L., L'analyse statistique des données en géographie, Alidade, RECLUS, 268 p.

\section{Revues:}

Mappemonde $\mathrm{N}^{\circ}$ 4/1986. $\mathrm{N}^{\circ}$ spécial, Chorèmes et modèles, (GIP RECLUS).

\section{Atlas :}

FERRAS R., Atlas d'Espagne, Fayard / RECLUS, 96 p.

THERY H., Atlas du Brésil, Fayard / RECLUS, 80 p.

\section{RÉSUMÉS}

Les cartes, sous toutes leurs formes, sont l'outil essentiel du géographe. Elles servent à chercher, à comprendre, à argumenter et à communiquer les formes d'organisation spatiale de la vie des sociétés. Elles peuvent être belles. Elles doivent être justes.

All types of maps are the essential kit for a geographer. They help to find, to understand, to argument and to communicate the different forms of spatial organization of life in society. They can be beautiful. They must be accurate.

INDEX

Mots-clés : Carte, cartographe, géographe

Keywords : geograph, Map

\section{AUTEUR}

GEORGES ROQUES

GIP RECLUS, maison de la géographie de Montpellier 